Conclusions SSFD successfully treated three patients with cervical carotid pseudoaneurysms at our institution from 2019 to present with no complications, satisfactory angiographic appearance, and symptom resolution. Though further research is required to confirm these results, we demonstrate that SSFD appears both safe and effective for endovascular treatment of cervical carotid pseudoaneurysms.

Disclosures R. Achey: None. L. Sheikhi: None. T. Patterson: None. G. Toth: None. N. Zobenica Moore: None. M. Bain: None.

\section{E-120 THE UTILITY OF REPEAT COMPUTED TOMOGRAPHY ANGIOGRAM AFTER BLUNT CEREBROVASCULAR INJURY}

Z Aljuboori ${ }^{\star}, \mathrm{K}$ Meyer, R James, D Ding. Neurosurgery, University of Louisville, louisville, KY

\subsection{6/neurintsurg-2020-SNIS. 152}

Introduction Blunt cerebrovascular injury (BCVI) can lead to thromboembolic events. The necessity of short-term repeat vascular imaging after the initial diagnosis is controversial. The aim of this retrospective cohort study is to assess the utility of short-interval computed tomography angiography (CTA) after an initial diagnosis of BCVI.

Methods We retrospectively reviewed consecutive patients with BCVI managed at the University of Louisville from 2016-2019 who underwent short-term (1-3 weeks) repeat CTA after initial diagnosis. The exclusion criteria were age $<18$ years, penetrating injury, and previous neck irradiation. We collected baseline data and performed logistic regression analysis to identify predictors of BCVI imaging outcomes.

Results The study cohort comprised 38 patients (68\% male) with a mean age of 45 years. Motor vehicle accident (79\%) was the most common mechanism of injury, and $89 \%$ had cervical spine fractures. Unilateral VA and grade I dissection were the most common findings on initial CTA were a unilateral VA dissection (66\%) that was Biffl grade I (36\%). Antiplatelet therapy or anticoagulation was administered to $82 \%$ of patients after the initial diagnosis (table 1). Shift analysis showed a significant improvement in Biffl grades on repeat CTA $(p=0.0001)$ (figure 1$)$. Biffl grades I injuries were more likely to improve (relative risk ratio $[R R R]=3.6$, CI $95 \%$ $(1.02-13.1), \mathrm{p}=0.04)$, whereas grade IV injuries were more

\begin{tabular}{lll} 
Abstract E-120 Table 1 & Patients demographics & \\
\hline Variable & Percentage \\
\hline Gender & Males & $68 \%$ \\
Mechanism of injury & Fall & $21 \%$ \\
MVA & $79 \%$ & \\
Cervical spine fractures & $89 \%$ \\
First rib fractures & $16 \%$ \\
Early Stroke & $5 \%$ \\
Delayed Stroke & $0 \%$ \\
Vascular intervention & $8 \%$ \\
Received ASA or AC between 1st and 2nd CTA & $82 \%$ \\
Received ASA or AC after 2nd CTA & $82 \%$ \\
Abbreviations: AC; anticoagulation, ASA; aspirin, CTA; computed & \\
tomography angiogram, MVA; motor vehicle accident, FFH; fall from & \\
height & \\
\hline
\end{tabular}

likely to be stable $(\mathrm{RRR}=33$, CI 95\% (2.9-374), $\mathrm{p}=0.005)$. Ten BCVIs (26\%) completely resolved on repeat imaging (table 2). The rates of early ( $<2$ weeks) and delayed ( 2 weeks to 3 months) ischemia were $5 \%$ and $0 \%$, respectively. Endovascular stenting was performed in $8 \%$.

Conclusion Short-term repeat non-invasive vascular imaging can help to identify the evolution of BCVI. Repeat vascular imaging at short intervals can help to identify patients who may require endovascular intervention, but additional studies are necessary to clarify its role in the management of BCVI. Disclosures Z. Aljuboori: None. K. Meyer: None. R. James: None. D. Ding: None.

\section{E-121 SALVAGE ENDOVASCULAR STRATEGIES IN A UNIQUE DUPLICATED INTERNAL CAROTID ARTERY LEADING TO CATASTROPHIC DISSECTION AND FUSIFORM ANEURYSM}

Y Lodi*, B Pulgarin, Z Weiss, S Chin, V Reddy. Neurology, Neurosurgery and Radiology, Upstate Medical University/UHS-Wilson Medical Center, Johnson City, NY

10.1136/neurintsurg-2020-SNIS.153

Background Anatomical variance of duplicated internal carotid artery (ICA) is novel. However, duplication associated 
dissecting stenosis in one lumen and fusiform aneurysm in second lumen is not described.

Objective To report a case of ICA dissection originating from a duplicated ICA that caused an intracranial occlusion and extracranial fusiform aneurysm. Additionally, we report our endovascular salvage strategies.

Case A 41-year-old woman with history of hypertension and migraines presented with worsening headaches. Angiography demonstrated left ICA dissection without critical stenosis, but small pseudoaneurysm, patient was managed conservatively. Follow-up MRA required for worsening symptoms, showed deteriorating dissecting-narrowing at the skull-base in the petrous ICA. Emergent angiography revealed a dissecting long segment lesion extending from the distal cervical carotid artery to the petrous segment with critical stenosis of $90 \%$. Duplicated ICA originated $20 \mathrm{~mm}$ from the ICA origin and ended at C2 level meeting the original ICA from where the dissection began. The other duplication demonstrated a fusiform dilation at the center. Petrous ICA was repaired by PTA followed by Neuroform EZ stent and distal cervical ICA was reconstructed with additional 3 Neuroform Allas stents. Despite reconstruction, antegrade flow to the ICA remained slow due to the competitive flow from other duplications. Emergent shut-down of the fusiform duplication obtained by embolizing 3 large Stryker XL coils resulting in restoration of antigrade flow to the stented segments. Patient remained neurologically intact and discharged home in 2 days.

Conclusions Carotid duplication may lead to dissection and fusiform aneurysm formation. Competitive flow from duplication may responsible for dissection. Sacrifice of one of the duplication of the ICA, especially, the fusiform one is needed to maintain persistent antigrade flow.

Disclosures Y. Lodi: None. B. Pulgarin: None. Z. Weiss: None. S. Chin: None. V. Reddy: 2; C; Terumo. 3; C; Janssen, Chiesi Inc, Portola.

\section{E-122 YTTRIUM-90 RADIOEMBOLIZATION AS A POSSIBLE NEW TREATMENT FOR BRAIN CANCER: PROOF OF CONCEPT AND SAFETY ANALYSIS IN A CANINE MODEL}

${ }^{1} S$ Manupipatpong*, ${ }^{1} \mathrm{~A}$ Pasciak, ${ }^{2} \mathrm{~F}$ Hui, ${ }^{3} \mathrm{R}$ Krimins, ${ }^{4} \mathrm{~L}$ Gainsburg, ${ }^{5} \mathrm{M}$ Dreher, ${ }^{6} \mathrm{D}$ Kraitchman, ${ }^{1} \mathrm{C}$ Weiss. ${ }^{1}$ Interventional Radiology, Johns Hopkins University School of Medicine, Baltimore, MD; ${ }^{2}$ Neurointerventional Radiology, Johns Hopkins University School of Medicine, Baltimore, MD; ${ }^{3}$ Anesthesiology and Critical Care Medicine; Radiology and Radiological Science, Johns Hopkins University, Baltimore, MD; ${ }^{4}$ Mid-Atlantic Veterinary

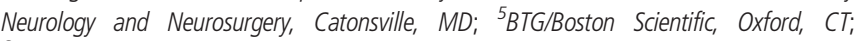
${ }^{6}$ Radiology and Radiological Science, Johns Hopkins University, Baltimore, MD

10.1136/neurintsurg-2020-SNIS.154

Introduction/Purpose Glioblastoma multiforme is the most common and aggressive type of brain tumor, with a median survival time of 15 months despite treatment. We propose the use of Y-90 endovascular radiosurgery (ER) to increase treatment efficacy while reducing the neurotoxicity associated with radiotherapy in glioblastoma. To that end, this study aimed to evaluate the safety and feasibility of Y-90 ER in the treatment of glioma in a canine model.

Materials and Methods Three healthy research dogs (R1-3) and five client-owned dogs on anti-epileptics with spontaneous, intra-axial brain masses (P1-5) received unilateral Y-90 glass microsphere infusions in either the PCA (R1), MCA (R2), or ICA (R3, P1-5), followed by quantitative Y-90 PET/
CT. R1-3 had neurological exams, as clinically indicated, and a 4-week post-ER MRI. P1-4 had serial neurological exams and 1-, 3-, and 6-month MRIs. Due to a small sample size, only descriptive statistics are reported.

Results R2-3 developed transient neurologic defects consistent with the treated side, which resolved in 13 days. R1 had no post-procedure neurologic deficits. The treated hemisphere in R1-3 received a maximum of $378 \pm 121 \mathrm{~Gy} \quad(\mathrm{x} \square \pm \boldsymbol{\sigma}$ ) of radiation, with maximum dose twice as high in more distal deliveries (PCA, MCA). MRIs at 1 month were normal without atrophy or microinfarction.

All dogs except P2 were on corticosteroids and seizure-free prior to treatment. P1-3 had transient post-procedure neurologic deficits which resolved in 20-33 days, while P4 had no neurologic deficits. P5 passed away 12-hours post-ER. The masses received $46.2 \pm 11.7 \mathrm{~Gy}$ of radiation with $19.3 \pm 12.2 \%$ of the mass volume receiving $>70 \mathrm{~Gy}$. MRIs at 1-month postER showed decreased mass size in all four dogs: by $69 \%$ and $59 \%$ in $\mathrm{P} 1$ and $\mathrm{P} 2$ on post-contrast MRI and by $24 \%$ and $26 \%$ in P3 and P4 on FLAIR MRI. The average number of spheres per $\mathrm{cm}^{3}$ of lesion, calculated using the measured activity per $\mathrm{cm}^{3}$ on PET/CT and expected activity per bead, ranged from 1490 (P2) to 5280 (P1).

At 53 days, P2's seizures returned with tumor enlargement, and he was euthanized 5 months post-ER. P1 remained asymptomatic until her 6-month visit, when left rear limb proprioceptive delay was observed. At her 12-month follow-up, left thoracic limb proprioceptive delay was also observed, though this did not suggest significant change in the known right cerebral cortex lesion. P3 remained asymptomatic with stable disease at his 6-month visit. P4 developed medically manageable seizure activity and a unilateral menace deficit with no mass growth on MRI at 3 months post-ER, though at 6 months mass volume began trending towards pre-treatment size.

Conclusion Y-90 ER in the canine brain is technically feasible and caused no permanent neurologic deficit despite $>400 \mathrm{~Gy}$ of radiation to critical brain structures. Four of five patient dogs had favorable dosimetric, radiologic, and clinical outcomes, all outliving the 63-day mean survival time associated with their original diagnosis and symptomatic treatment. Long-term outcomes, histopathology, and a larger sample size are needed to better understand brain Y90 ER viability.

Disclosures S. Manupipatpong: None. A. Pasciak: None. F. Hui: None. R. Krimins: None. L. Gainsburg: None. M. Dreher: 5; C; BTG/Boston Scientific. D. Kraitchman: None. C. Weiss: 1; C; BTG/Boston Scientific. 2; C; BTG/Boston Scientific.

\section{E-123 MIDDLE MENINGEAL ARTERY EMBOLIZATION FOR CHRONIC SUBDURAL HEMATOMAS: AN INSTITUTIONAL ANALYSIS}

J Catapano*, C Nguyen, M Pacault, T Cole, J Baranoski, D Wilkinson, N Majmundar, A Ducruet, F Albuquerque. Neurosurgery, BNI, Phoenix, AZ

\subsection{6/neurintsurg-2020-SNIS. 155}

Introduction Chronic subdural hematoma (cSDH) is one of the most common neurological diseases, often causing significant morbidity especially in the elderly population. Recently, middle meningeal artery (MMA) embolization has emerged as 